\title{
Estımation of Absorbed Dose Distribution in Different Organs during the CT Scan: Monte Carlo Study
}

\author{
Umit Kara ${ }^{1 *}$ and Huseyin Ozan Tekin ${ }^{2}$ \\ ${ }^{1}$ Medical Imaging, Suleyman Demirel University, Turkey \\ ${ }^{2}$ Department of Radiotherapy, Uskudar University, \\ Turkey \\ *Corresponding author: Umit Kara, Suleyman \\ Demirel University, Vocational School of Health Services, \\ Medical Imaging, 32100, Isparta, Turkey
}

Received: March 08, 2017; Accepted: March 30, 2017; Published: April 04, 2017

\begin{abstract}
This work aimed to validate the accuracy of a Monte Carlo source model of the Ge Lightspeed CT scanner using organ doses measured in specific human adult phantoms. The x-ray output of the Ge Lightspeed multidetector CT scanner was simulated within the Monte Carlo code. The resulting source model was able to perform various real simulated scan model helical Computed Tomography (CT) scans of varying scan parameters such as kVp, mAs, filtration, pitch, and beam collimation. This work has been performed by using real Computed Tomography (CT) protocols to patients in public hospitals in Turkey. We used Monte Carlo simulation methods with real height and weight of patients and Computed Tomography (CT) scanners real parameters. Absorbed organ doses have been calculated by using Monte Carlo simulation. The results showed that changes in $\mathrm{mAs}$ value are significantly important for obtaining the risk of cancer from dose rates. Additionally, the dose received by each organ has been calculated and the results showed that Monte Carlo is a strong and effective tool in radiological investigations.
\end{abstract}

Keywords: CT scan; Absorbed dose; Monte carlo simulation

\section{Introduction}

Diagnostic radiology is a significant tool for clinical diagnosis and includes general x rays, Computed Tomography (CT), Magnetic Resonance Imaging (MRI), ultrasound, mammography, etc. Diagnostic radiology is part of medicine that uses medical imaging facility and technology to diagnose disease and helps define the structures inside in human body. Diagnostic radiology uses the imaging technologies of X-rays radiography, computed tomography, magnetic resonance imaging, ultrasound, mammography, etc. Common classifications of CT scans are abdominal, bone, head and vascular system. CT scanning is the one of most used units in diagnostic radiology and these units combine the use of X-rays and computer processing to generate tomographic images of the body. The use of CT in radiology and medical imaging has been growing in the world. CT is useful as it allows the radiologist, to view a crosssectional picture of the entire body. CT is painless, fast, and accurate and CT scan uses $\mathrm{x}$-ray equipment and computers to produce medical images that often can make more detailed image than conventional radiography. CT scans use ionizing radiation as used in other $\mathrm{x}$-rays units. In clinical, the benefits of a correct diagnosis preponderance to the risk of exposure to ionizing radiation during the body scan. In diagnostic radiology, it is relevant to see the absorbed doses in organs, particularly those that receive the highest radiation doses. Absorbed radiation doses are estimated using standardized reference. The Monte Carlo (MC) simulation has been widely adopted to medical physics and medical imaging. In a medical physics simulation estimated image of the conditions is modeling to a computer program, which then authorizes imaginary radiation particles, waves and simulates interactions with the program tools. Although there are a variety of methods by which one can estimate patient organ doses from CT examinations, Monte Carlo simulations have been reported to be the most accurate, reliable, and versatile in accomplishing this task [1-8].

In the Monte Carlo method, the patient and CT scanner are simulated using a computational anatomic model of the patient and an $\mathrm{x}$-ray source model representing the scanner's beam output. However, to ensure the accuracy of these calculations, these CT source models must be benchmarked and validated against actual experimental measurements made on the scanners they simulate. In the past, most validation studies were accomplished using standard CT Dose Index (CTDI) phantoms, but in recent years, anthropomorphic phantoms have been increasingly utilized $[9,10]$. Mathematical phantoms or MIRD phantoms have been produced [11] which were the first models of human phantoms to be widely used in dosimetry studies involving X-ray exposure by the Monte Carlo method.

However, the organs in these phantoms are described by mathematical equations with limited representation of the actual structure of a human body and its chemical and physical characteristics [12]. Voxel-based phantoms created from tomography images present a geometry which adequately represents a patient, including internal organ, displacements, and deformations. These phantoms are recommended for dosimetric studies with the Monte Carlo method because more consistent results are obtained with adult reference computational phantoms, as described in ICRP $110[13,14]$. This study aimed to investigate the using availability of Monte Carlo technique during the calculation of absorbed dose amounts by different organs and make an assessment on cancer risk by considering dose magnitudes.

\section{Materials and Methods}

A General Electric (GE) Lightspeed multidetector CT scanner was used as the base for the Monte Carlo code model as well as for all radiation dose and organ absorbed dose measurements. The 


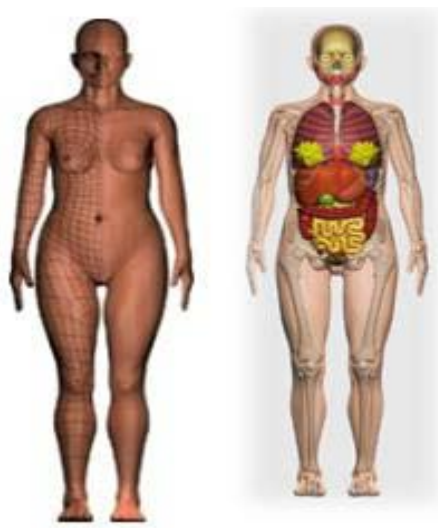

Figure 1: Human Body Phantoms.

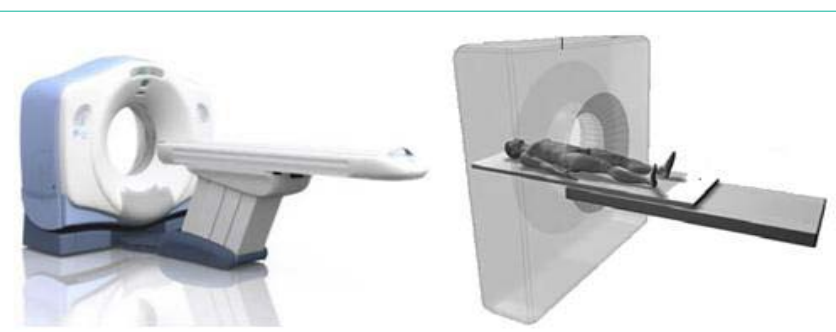

Figure 2: Source Model of the CT Scanner.

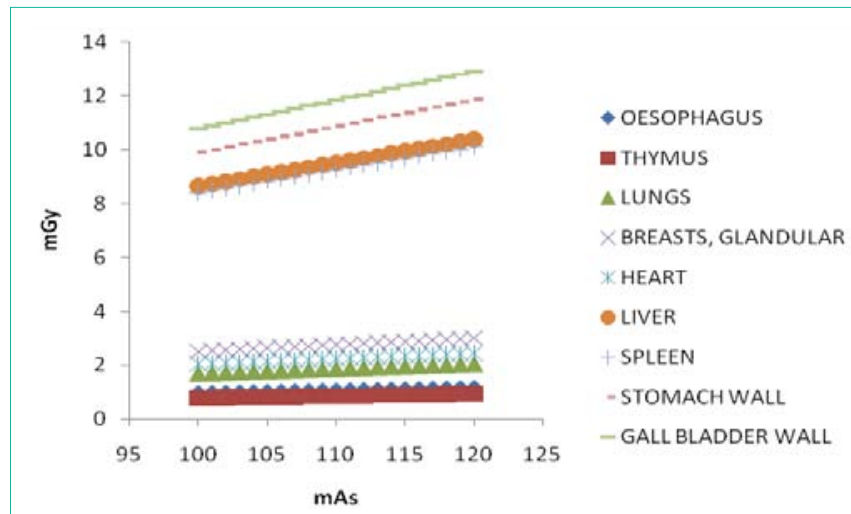

Figure 3: Absorbed organ dose (mGy/100-120 mAs) for phantom.

GE Lightspeed multidetector CT scanner was measured with real protocols to patients in public hospitals and used Monte Carlo simulation methods with the real height and weight of patients and the CT scanner's actual parameters (Table 1). Absorbed organ doses were then calculated based on these Monte Carlo results. Monte Carlo simulation in the CT scanner containing 64 rows of detectors, scan mode helical, total collimation $40.0 \mathrm{~mm}$, table height $133.00 \mathrm{~mm}$, pitch 1.53, filter $10.0 \mathrm{~mm} \mathrm{Al} 7$ Deg Tungsten, mean spectral energy $64.9 \mathrm{keV}$, helical scan table increment $6 \mathrm{~cm}$, number of rotations 6 , source-to-iso-centre distance $53.9 \mathrm{~cm}$, total field size at iso-centre $57.3 \mathrm{~cm} \mathrm{x} 36.0 \mathrm{~cm}$, beam width at iso-centre $4 \mathrm{~cm}$, scanlength $36 \mathrm{~cm}$ (Table 2).

The operator selected tube potentials of $120 \mathrm{kVp}$ along tube current and gantry rotation speeds and tube current of 100-120 mAs among with varying tube current. The scanner moreover makes use of

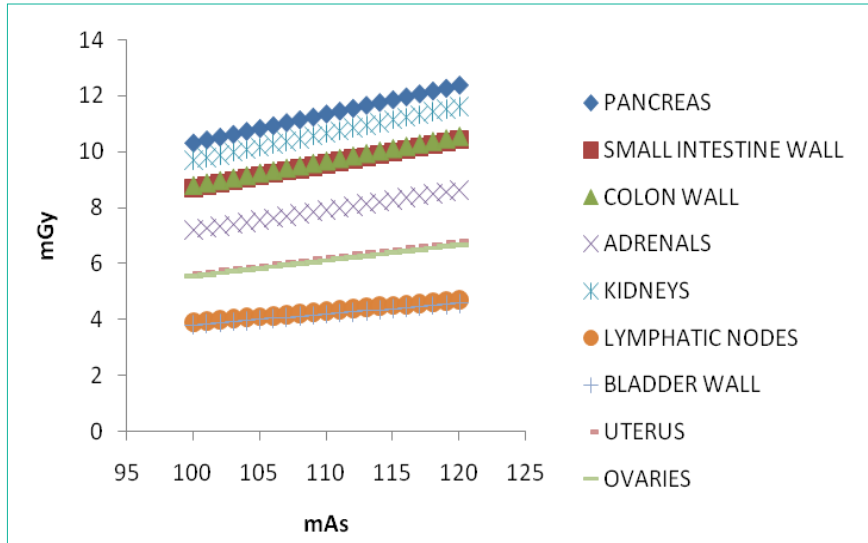

Figure 4: Absorbed organ dose (mGy/100-120 mAs) for phantom

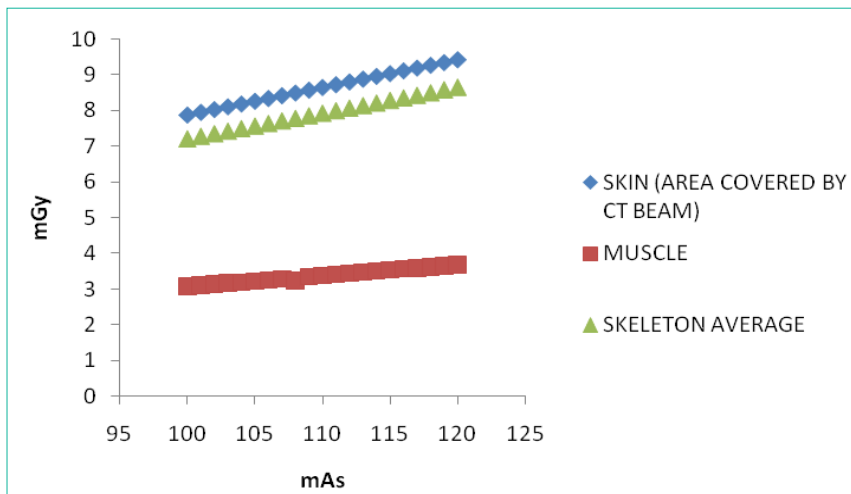

Figure 5: Absorbed organ dose (mGy/100-120 mAs) for skin, muscle and skeleton.

tube current modulation during scanning, this feature was used in the measurements important in this study. Tube current (milliamperes) is an importantdeterminant of radiation dose and image quality in CT examinations. The aim of work with DoseWatch tube current modulation (100-120 mAs) is to maintain constant image quality and showed radiation dose to patients to be reduced. Organ dose calculations using the Monte Carlo CT source model were performed using the human body voxel phantoms which can be seen in Figure 1. The source model of the CT scanner was used as a real source file within a general purpose Monte Carlo simulation code shown in Figure 2 (GE Lightspeed multidetector CT). Material and thickness data for the two inherent filters for abdomen/pelvis scanning were used with the CT unit. The Monte Carlo model allows the user to simulate both axial exams and helical scans but we used helical scan and real protocols in this exam. Helical exams are accomplished by having the source first sample the location of the $\mathrm{x}$-ray focal spot along a mathematically described helix based on the pitch and scan length selected with real protocol and measured, as well as the previously defined source to iso centre distance $53.9 \mathrm{~cm}$, Total field size at isocentre $57.3 \mathrm{~cm} \times 36.0 \mathrm{~cm}$, beam width at iso-centre $4 \mathrm{~cm}$. The photon energy was worked based on the mean spectral energy selected 64.9 $\mathrm{keV}$ for the scan. This process was repeated for the same patient and different tube current to be transported (100-120 mAs).

\section{Results and Discussion}

CT uses ionizing radiation, the radiographer, radiology 
Table 1: Clinical CT Scan parameters.

\begin{tabular}{|c|c|c|c|c|c|c|c|c|c|c|c|c|}
\hline Age & Gender & CT scan & mAs & kV & Ctdivol & $\begin{array}{l}\text { Dip (mgy } \\
\text { cm) }\end{array}$ & Total dlp & Bmı & Scan mod & $\begin{array}{c}\text { Total } \\
\text { collimation }\end{array}$ & Table heıght & Pitch* \\
\hline 21 & $\begin{array}{c}\text { Female Adult } \\
\text { Patıent }\end{array}$ & Abdomen/Pelvis & $0-120$ & 120 & .01 & 49.63 & 149.63 & 23.14 & HELICAL & $40.0 \mathrm{~mm}$ & $133.00 \mathrm{~mm}$ & 1.53 \\
\hline
\end{tabular}

Table 2: CT Scan parameters used in Monte Carlo simulation.

\begin{tabular}{|c|c|c|c|c|c|c|c|c|c|c|c|}
\hline Age & Gender & CT scan & Mas & Kv & $\begin{array}{l}\text { Body } \\
\text { mass }\end{array}$ & $\begin{array}{l}\text { Standing } \\
\text { height }\end{array}$ & Filter & $\begin{array}{l}\text { Mean spectral } \\
\text { energy }\end{array}$ & $\begin{array}{l}\text { Helıcal (spiral) scan, } \\
\text { table increment }\end{array}$ & $\begin{array}{l}\text { Number of } \\
\text { rotations }\end{array}$ & Pitch* \\
\hline 21 & $\begin{array}{c}\text { Female } \\
\text { Adult } \\
\text { Patıent }\end{array}$ & $\begin{array}{l}\text { Abdomen } \\
\text { Pelvis CT }\end{array}$ & $100-120$ & 120 & $71.8 \mathrm{Kg}$ & $172.2 \mathrm{~cm}$ & $\begin{array}{l}10.0 \mathrm{~mm} \\
\text { Al } 7 \mathrm{Deg} \\
\text { Tungsten }\end{array}$ & $64.9 \mathrm{keV}$ & $6 \mathrm{~cm}$ & 6 & 1.53 \\
\hline Age & Gender & CT scan & Mas & Kv & $\begin{array}{l}\text { Body } \\
\text { mass }\end{array}$ & $\begin{array}{l}\text { Standing } \\
\text { height }\end{array}$ & Filter & $\begin{array}{l}\text { Source-to-iso- } \\
\text { centre distance }\end{array}$ & $\begin{array}{c}\text { Total field size at iso- } \\
\text { centre }\end{array}$ & $\begin{array}{l}\text { Beam width at } \\
\text { iso-centre }\end{array}$ & $\begin{array}{l}\text { Scan } \\
\text { length }\end{array}$ \\
\hline 21 & $\begin{array}{c}\text { Female } \\
\text { Adult } \\
\text { Patıent }\end{array}$ & $\begin{array}{l}\text { Abdomen } \\
\text { Pelvis CT }\end{array}$ & $100-120$ & 120 & $71.8 \mathrm{Kg}$ & $172.2 \mathrm{~cm}$ & $\begin{array}{c}10.0 \mathrm{~mm} \\
\mathrm{Al} 7 \mathrm{Deg} \\
\text { Tungsten }\end{array}$ & $53.9 \mathrm{~cm}$ & $57.3 \mathrm{~cm} \mathrm{x} 36.0 \mathrm{~cm}$ & $4 \mathrm{~cm}$ & $36 \mathrm{~cm}$ \\
\hline
\end{tabular}

technologist should use their equipment and protocols to keep doses "patient-sized." CT technology works hard to keep doses as low as possible without compromising the image quality needed to make a correct diagnosis The standard unit of measure for radiation absorbed by an individual is called the "Sievert," or Sv and sometimes identified by a smaller unit called the "millisievert," or mSv. Common medical imaging tests such as X-rays or mammograms generally expose patients to a radiation dose of less than $1 \mathrm{mSv}$. Other procedures using CT, nuclear stress tests, or fluoroscopy-guided exams often involve radiation in the range of $5-40 \mathrm{mSv}$. New advances in CT technology, like GE Healthcare's ASIR, have helped reduce the typical range of radiation for CT exams to 1-15 mSv. Radiation can also be expressed in the unit Gray (Gy), a measurement of the absorbed dose of radiation. This term replaces a former unit of measurement called the rad. A single exposure at these diagnostic levels may not pose much risk to the patient. When a patient has numerous tests over a period of time, the cumulative exposure may raise the level of risk. To minimize cumulative exposure, physicians should determine whether a procedure using medical radiation is necessary to achieve the diagnosis or whether an alternative imaging procedure may offer the same diagnostic benefit. In this study, a simulation geometry has been generated by using the Monte Carlo method to calculate the abosrbed radiation dose amounts in critical organs during the Abdominal/ Pelvis scan. As it can be seen in Figure $3 \& 4 \& 5$, increasing $\mathrm{mAs}$ value has been caused the increasement of aborobed dose amount in different organs. The results showed that each increase rate has been caused to increasement of absorbed dose rate in critical organs which remaining in the both scan area and peripheral regions. In summary, it is important and necessary to adapt and follow scan protocols. For this aim, each possible method for dose reduction should be tried.

\section{Conclusion}

In present study, the absorbed dose amounts for abdominal/pelvis scan have been investigated. It was found that each increase of $\mathrm{mAs}$ value in scan has been caused an increase in the amount of absorbed dose amount in critical organs. The results indicated that working at low $\mathrm{mAs}$ values and appling the dose protocols are significant. Due to the radiosensitivity of ciritical organs, it is obvious that increasement of $\mathrm{mAs}$ value will cause a direct effect to short and long term biological effects.. It can be also concluded that investigated standart Monte Carlo geometry can be used for potential future studies since absorbed dose investigations recently used for dose optimizationin CT applications.

\section{References}

1. Tekin H.O. MCNP-X Monte Carlo Code Application for Mass Attenuation Coefficients of Concrete at Different Energies by Modeling $3 \times 3$ Inch Nal(TI) Detector and Comparison with XCOM and Monte Carlo Data. Science and Technology of Nuclear Installations. 2016.

2. Akkurt I, Tekin HO, Mesbahi A. Calculation of Detection Efficiency fort he Gamma Detector using MCNP-X. Acta Physica Polonica A. 2015; 128: 332334.

3. Tekin HO, Kara U. Monte Carlo Simulation for Distance and Absorbed Dose Calculations in a PET-CT Facility by using MCNP-X. Journal of Communication and Computer. 2016; 13: 32-35.

4. Tekin HO, SinghVP, Kara U, Manici T, Altunsoy EE. Investigation of Nanoparticle Effect on Radiation Shielding Property Using Monte Carlo Method. CBU Journal of Science. 2016; 12.

5. Tekin HO, Singh VP, Manici T. An Investigation on Shielding effect of Bismuth on Lung CT Scan using Monte Carlo Simulation. Journal of Polytechnic. 2016; 19: 617-620.

6. Tekin HO, Singh VP, Manici T. Effects of micro-sized and nano-sized WO3 on mass attenuation coefficients of concrete by using MCNPX code. Applied Radiation and Isotopes. 2016.

7. DeMarco JJ, Cagnon CH, Cody DD, Stevens DM, McCollough CH, Daniel $\mathrm{JO}$, et al. A Monte Carlo based methodto estimate radiation dose from multidetector CT (MDCT): Cylindrical andanthropomorphic phantoms. Phys Med Biol. 2005; 50: 3989-4004.

8. Liu H, Gu J, Caracappa PF, Xu XG. Comparison of two types ofadult phantoms in terms of organ doses from diagnostic CT procedures. Phys Med Biol. 2010; 55: 1441-1451.

9. Deak P, van Straten M, Shrimpton PC, Zankl M, Kalender WA. Validation of a Monte Carlo tool for patient-specific dose simulations inmulti-slice computed tomography. Eur. Radiol. 2008; 18: 759-772.

10. Li X, Samei E, Segars WP, Sturgeon GM, Colsher JG, Toncheva G, et al. Patient-specific radiation dose and cancerrisk estimation in CT: Part I. Development and validation of a MonteCarlo program. Med Phys. 2011; 38: 397-407.

11. Jarry G, DeMarco J, Beifuss U, Cagnon CH, McNitt-Gray MF. A Monte Carlobased method to estimate radiation dose from spiral CT: from phantom testing to patient-specific models. Physics in Medicine and Biology. 2003; 48: 2645.

12. Olsher RH, Riper KAV. Application of a sitting MIRD phantom for effective dose calculations. Radiation Protection Dosimetry. 2005; 116: 392.

13. Lee C, Lee C, Staton RJ, Hintenlang DE, Arreola MM, Williams JL, et al. Organ and effective doses in pediatric patients undergoing helical multislice computed tomography examination. Medical Physics. 2007; 34: 1858-1873.

14. Adult Reference Computational Phantoms. ICRP Publication 110. Ann ICRP $2009 ; 39$. 